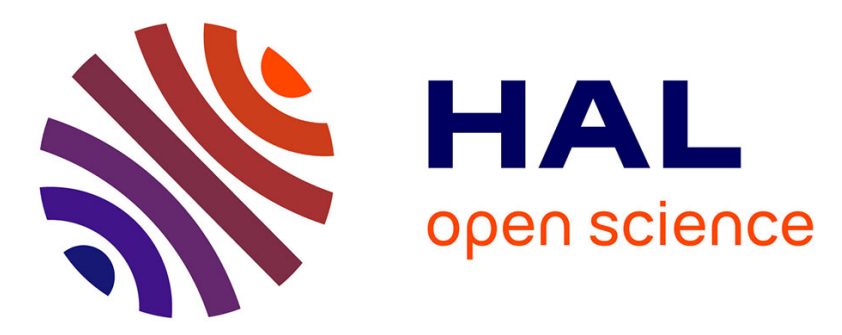

\title{
Application de la théorie linéaire de la mécanique de la rupture aux structures métalliques épaisses - Méthodes pratiques de calcul des facteurs d'intensité de contrainte
}

R. Labbens, A. Pellissier-Tanon, J. Heliot

\section{- To cite this version:}

R. Labbens, A. Pellissier-Tanon, J. Heliot. Application de la théorie linéaire de la mécanique de la rupture aux structures métalliques épaisses - Méthodes pratiques de calcul des facteurs d'intensité de contrainte. Revue de Physique Appliquée, 1974, 9 (4), pp.587-598. 10.1051/rphysap:0197400904058700 . jpa-00243822

\section{HAL Id: jpa-00243822 \\ https://hal.science/jpa-00243822}

Submitted on 1 Jan 1974

HAL is a multi-disciplinary open access archive for the deposit and dissemination of scientific research documents, whether they are published or not. The documents may come from teaching and research institutions in France or abroad, or from public or private research centers.
L'archive ouverte pluridisciplinaire HAL, est destinée au dépôt et à la diffusion de documents scientifiques de niveau recherche, publiés ou non, émanant des établissements d'enseignement et de recherche français ou étrangers, des laboratoires publics ou privés. 


\title{
APPLICATION DE LA THÉORIE LINÉAIRE \\ DE LA MÉCANIQUE DE LA RUPTURE AUX STRUCTURES MÉTALLIQUES ÉPAISSES
}

\section{MÉTHODES PRATIQUES DE CALCUL DES FACTEURS D'INTENSITÉ DE CONTRAINTE $\left({ }^{*}\right)$}

\author{
R. LABBENS, A. PELLISSIER-TANON et J. HELIOT \\ CREUSOT-LOIRE, Branche mécanique et entreprise \\ 15, rue Pasquier, 75008 Paris, France
}

\begin{abstract}
Résumé. - On résume les fondements de la Théorie Linéaire de la Mécanique de la Rupture. On montre comment cette théorie peut être appliquée à des structures industrielles et non seulement à des géométries et des chargements simples, et comment les facteurs d'intensité de contrainte peuvent être définis dans tous les cas. On examine sous quelles conditions le facteur d'intensité de contrainte est un critère de rupture brutale, et les corrections et limitations qui résultent de la déformation plastique. On montre ensuite comment la connaissance des fonctions poids attachées à une géométrie ramène à une quadrature le calcul du facteur d'intensité de contrainte. On donne des fonctions de poids pour une longue fissure axiale et une fissure circonférentielle dans un cylindre de révolution.

Abstract. - The bases of Linear Elastic Fracture Mechanics are summed up. It is shown how this theory can be applied to industrial structures and not only to simple geometries and loads, and how stress intensity factors can always be defined. The conditions under which the stress intensity factor is a fast fracture criterion are examined, as well as corrections and limitations resulting from plastic deformation. It is shown how weight functions depending only on geometrical parameters make the calculation of the stress intensity factors possible by a simple integral, for any load applied to a given body. Such weight functions are given for a long axial crack and a circumferential crack in a cylinder of revolution.
\end{abstract}

En 1970-71, lorsque la recherche résumée ci-dessous fut organisée, il apparaissait que les développements sur la Mécanique de la Rupture avaient été en France surtout métallurgiques, que les exposés mécaniques qu'on trouvait portaient le plus souvent sur des problèmes plans, simples à la fois par la géométrie et le chargement. Or, n'importe quel problème de bureau d'études comporte des chargements assez complexes, tels que des contraintes thermiques, et des géométries assez variables : corps de révolution, concentrations de contrainte, etc... Les documents étrangers, américains surtout, qu'on pouvait se procurer, traitaient aussi surtout de problèmes de principe simples, et malgré des développements mathématiques parfois difficilement abordables, ne montraient pas clairement comment faire des applications pratiques un peu complexes.

$\left(^{*}\right)$ Résumé des recherches exécutées par CREUSOT-LOIRE au titre du contrat de la Délégation Générale à la Recherche Scientifique et Technique $n^{\circ}$ 71-7-2885.
L'idée directrice de la recherche faite à CREUSOTLOIRE, orientée vers la Grosse Mécanique et les fortes épaisseurs en matériaux de caractéristiques de traction moyennes, fut donc la suivante :

- dans les limites de validité de la théorie linéaire, rechercher si tous les problèmes mettant en jeu des géométries et des chargements quelconques pouvaient avoir une solution théorique ou pratique, et essayer de dégager des méthodes de calcul industriellement utilisables ;

- rechercher si des solutions existaient hors des limites de la théorie linéaire.

Le programme fut évidemment précisé en cours de travail, et on traita les questions suivantes :

- à l'intérieur de la théorie linéaire :

- hypothèse fondamentale ; critère d'instabilité ;

- forme très générale du champ singulier de contrainte au voisinage du front de fissure ;

- à quelles conditions le facteur d'intensité de contrainte est-il un critère d'instabilité ?; 
- validité de l'utilisation pour tous les problèmes du facteur critique d'intensité de contrainte $K_{\mathrm{IC}}$ mesuré sur des éprouvettes planes ;

- méthodes industrielles de calcul des facteurs d'intensité de contrainte ;

- limites et prolongements de la théorie linéaire ; possibilité de mesurer $K_{\mathrm{IC}}$ à l'aide de petites éprouvettes en grande déformation plastique ;

- utilisation des concepts de la théorie linéaire pour l'étude d'autres phénomènes que la rupture brutale.

On examine les conclusions auxquelles on est arrivé sur ces problèmes, sans donner les justifications complètes qu'on trouvera dans des notes plus détaillées ou dans les références citées.

1. Théorie élastique linéaire. - 1.1 HYPOTHÈSE FONDAMENTALE ; CRITÈRE D'INSTABILITÉ. — Griffith [12] traita le problème particulier d'un plan indéfini fissuré soumis à des tractions à l'infini (Fig. 1). Il énonça une condition globale d'instabilité, indépendante de toute hypothèse, et intéressant tout le corps fissuré ; dans un accroissement $\mathrm{d} a$ de la fissure, l'énergie libérée - $\mathrm{d} U$ est égale ou supérieure à l'énergie $\mathrm{d} \mathscr{C}$ mise en jeu par l'extension $\mathrm{d} a$.

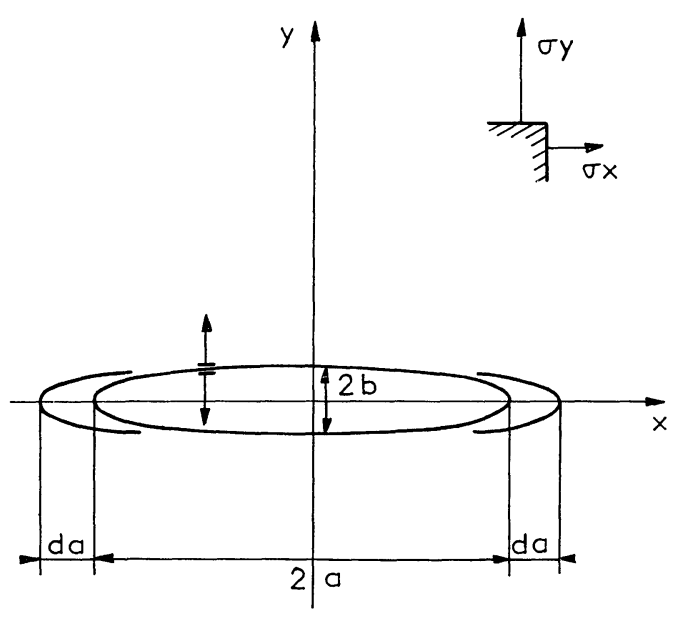

FIG. 1.

Il énonça ensuite l'hypothèse fondamentale de la théorie linéaire, hypothèse locale : $\mathrm{d} \mathscr{C}$ est proportionnel à l'extension de surface de fissure, $2 e \mathrm{~d} a$, et à une constante du matériau $\gamma$, qu'il crut pouvoir assimiler à l'énergie superficielle des liquides :

$$
\mathrm{d} \mathscr{C}=2 \times 2 \gamma e \mathrm{~d} a .
$$

D'où par un calcul simple la condition d'instabilité en déformation plane :

$$
a \sigma_{\mathrm{y}}^{2} \geqslant \frac{2 E \gamma}{1-v^{2}}
$$

Le second membre est une valeur critique, caractéristique du matériau.

D'autres hypothèses ont été faites sur l'énergie localement mise en jeu par l'extension de la fissure :

- dissipation par déformation plastique en avant du front de fissure (Irwin [14]-Orowan [22]) ;

- ruptures de liaisons atomiques (Barenblatt [2]).

Quelles que soient ces hypothèses, la théorie linéaire conserve :

$$
\mathrm{d} \mathcal{C}=\mathcal{G}_{\mathrm{c}} \mathrm{d} A
$$

$\mathcal{G}_{c}$ est, en déformation plane, une constante du matériau.

1.2 Champ De CONTRAinte Singulier AU voisinage DU FRONT DE FISSURE. - 1.2.1 Irwin [15-16] montra la généralité de la partie singulière du champ de contrainte et de la partie principale du champ de déplacement :

$$
\begin{aligned}
& \sigma_{i j}=\frac{K}{\sqrt{2 n^{r}}} f_{i j}(\theta) \\
& u_{i}=K \sqrt{\frac{2 r}{\pi}} g_{i}(\theta)
\end{aligned}
$$

la géométrie et le chargement n'ayant d'influence que sur le facteur d'intensité de contrainte $K$; avec trois modes de déplacement des faces de la fissure :

I. Ouverture,

II. Cisaillement dans le plan,

III. Cisaillement anti-plan.

La partie principale de l'énergie libérée dans une extension $\mathrm{d} a$ est :

$$
\begin{aligned}
-\mathrm{d} U=\mathscr{G} e \mathrm{~d} a & =\left(1-v^{2}\right) \frac{K^{2}}{E} e \mathrm{~d} a \quad \text { (déformation plane) } \\
& =\frac{K^{2}}{E} e \mathrm{~d} a \quad \text { (contrainte plane) }
\end{aligned}
$$

La considération de ce champ de contrainte singulier montre que la théorie linéaire conduit à une condition globale d'instabilité de la forme (1) ou d'une forme dimensionnellement équivalente pour toutes les géométries et tous les chargements.

Si le terme singulier est suffisant pour décrire le phénomène, il doit exister en déformation plane une valeur critique du facteur d'intensité de contrainte :

$$
K_{\mathrm{IC}}^{2}=\frac{E}{1-v^{2}} \mathcal{G}_{\mathrm{IC}}
$$

équivalente à la constante de Griffith, caractéristique mécanique du matériau.

La confirmation expérimentale fut bonne, surtout pour les matériaux à hautes caractéristiques de traction; en déformation plane $K_{\mathrm{IC}}$ dépend de la température et pour certains matériaux de la vitesse de mise en charge ; il existe une valeur d'arrêt d'une 
fissure en propagation rapide ; hors de la déformation plane, $K_{\mathrm{C}}$ dépend de la longueur de fissure et de l'éprouvette.

1.2.2 La validité des formules (3) fut étendue par Irwin [18] à une fissure elliptique soumise à une traction d'ouverture uniforme, avec un $K$ variable le long du front de fissure, et la déformation plane dans le plan normal. Sur la même géométrie, Kassir et Sih [19] montrèrent qu'un chargement plus général menait aux trois modes. Bueckner [7]-[9] traita le problème général d'une fissure plane quelconque, l'application pratique du calcul restant difficile.

On montre aussi qu'une singularité autre que $\frac{1}{2}$ conduit à $\mathcal{G}$ toujours infini si elle est plus grande, ou toujours nul si elle est plus petite; la singularité $\frac{1}{2}$ s'impose donc; il existe d'autres démonstrations.

La forme du champ singulier donnée par Irwin, avec les trois modes d'ouverture, est donc très générale.

1.2.3 Par le principe de superposition (Fig. 2), on montre que pour le calcul du champ singulier, tout chargement peut être remplacé par le chargement équivalent, obtenu en appliquant aux faces de la fissure des pressions opposées aux contraintes qui régneraient à l'emplacement de la fissure dans le corps non fissuré soumis au chargement extérieur.
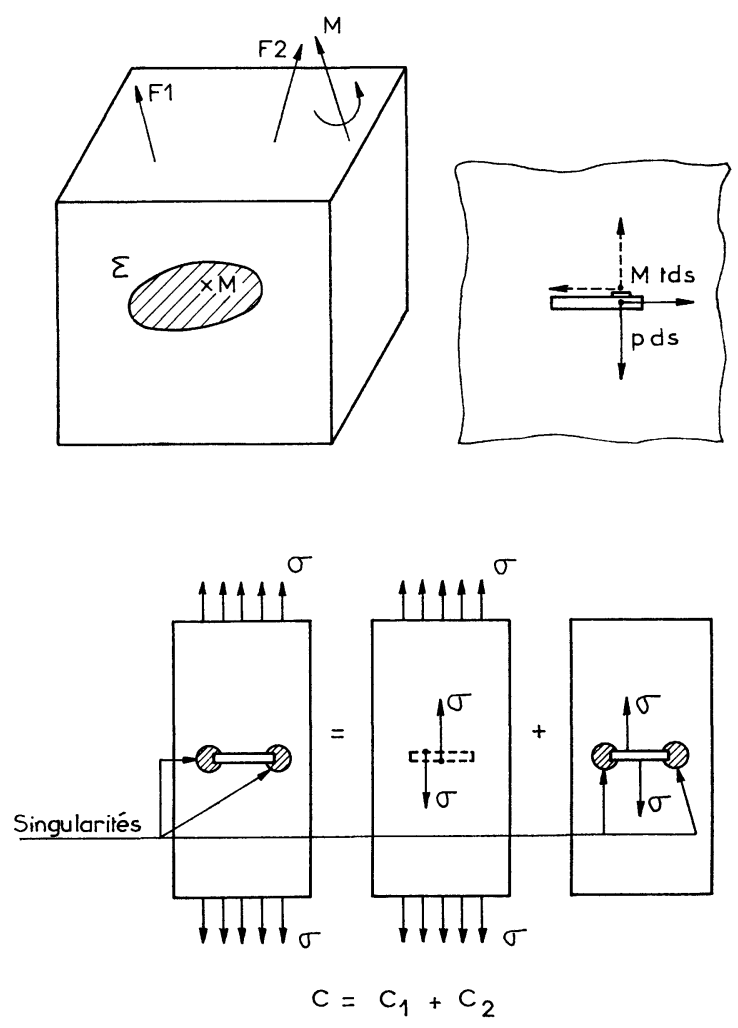

FIG. 2.

Bueckner [6] en a donné une démonstration rigoureuse par les théorèmes sur le travail virtuel.

Le calcul du champ singulier se ramène donc à un problème théoriquement simple.
1.3 Quand le facteur d'intensité de contrainte est-il un critère d'instabilité ?

1.3.1 Pour le calcul de l'énergie libérée, on a admis au par. 1.2.1 que l'extension de la fissure se faisait dans la direction de celle-ci, et que $K$ était constant le long du front de fissure, et on a calculé cette énergie en fermant l'extension par application des contraintes qui régnaient avant l'extension. On a trouvé alors l'expression (4).

Si la fissure a un contour quelconque, avec un $K$ variable, mais se prolonge toujours dans son plan, le calcul de l'énergie libérée devient :

$$
-\mathrm{d} U=\frac{1-v^{2}}{E} \int_{\mathrm{L}} K_{\mathrm{I}}^{2}(s) \mathrm{d} n \mathrm{~d} s .
$$

Il est plus difficile car il suppose une hypothèse sur $\mathrm{d} n$.

Si l'extension de la fissure ne se fait pas dans le plan de celle-ci, en général on ne sait calculer ni l'ouverture ni l'énergie libérée par l'extension; ce problème difficile est actuellement l'objet de recherches.

Il en résulte que le calcul de l'énergie libérée n'est simple que si la fissure prolonge dans son plan et si $K$ est constant sur le front de la fissure. Alors :

$$
K=K_{\mathrm{c}}
$$

est le critère de rupture. C'est le cas des problèmes plans et de révolution.

1.3.2 En mode I, par raison de symétrie, l'extension prolonge la fissure, et le calcul de l'énergie libérée est simple; mais en mode II ou sous un chargement complexe, il n'y a aucune raison pour que l'extension prolonge la fissure, et on est pratiquement désarmé.

On admet souvent que les modes II et III sont pratiquement sans grande importance parce que, pour les aciers au moins, les aspérités résultant du mode d'ouverture qui existe au voisinage du front de fissure gênent les glissements; le mode I agissant alors seul, on admet aussi que, au moins au début de la propagation, la direction moyenne d'extension prolonge la fissure. Lorsque $K_{\mathrm{I}}$ est constant, le critère :

$$
K_{\mathrm{I}}=K_{\mathrm{IC}}
$$

est donc rigoureux en mode $I$, approché en chargement mixte. D'ailleurs, on ne connaît pas de $K_{\text {IIC }}$ et $K_{\text {IIIC }}$.

Lorsque $K$ est variable sur la fissure, on admet souvent comme critère pessimiste :

$$
K_{\max }=K_{\mathrm{IC}}
$$

qui se comprend facilement si la variation de $K$ est faible ; si la variation est plus forte, l'énergie libérée, proportionnelle à $K^{2}$, est concentrée dans les régions de $K$ élevé, et l'approximation semble encore raisonnable.

C'est le cas de la plupart des problèmes tridimensionnels pour lesquels ce critère approché ne peut être utilisé qu'avec circonspection et jugement. 
1.3.3 La formulation d'un critère d'instabilité sous l'action de sollicitations provoquant les trois modes fait actuellement l'objet de recherches ; plusieurs hypothèses ont été avancées, mais ne sont pas encore clairement justifiées.

1.4 Le facteur critique d'intensité de contrainte $K_{\text {IC }}$ mesuré sur des éprouvettes en déformation plane peut-il être validement utilisé dans n'importe quel problème ? (de Leiris [20-21]).

On pouvait se demander si l'existence d'une contrainte parallèle au front de fissure, la contrainte circonférentielle sur une fissure circulaire dans un cylindre sous pression, ne créait pas une triaxialité plus sévère que celle qui existe dans l'éprouvette A. S. T. M. qui n'est pas chargée sur ses côtés.

En un point d'une fissure située dans le plan xoy, les composantes du tenseur des contraintes résultant du chargement sur le solide non fissuré et celles de la normale sont :

$$
\left\|\begin{array}{lll}
\sigma_{x x} & \tau_{x y} & \tau_{x z} \\
\tau_{y x} & \sigma_{y y} & \tau_{y z} \\
\tau_{z x} & \tau_{z y} & \sigma_{z z}
\end{array}\right\| \text { et }\left\|\begin{array}{l}
0 \\
0 \\
1
\end{array}\right\| \text {. }
$$

Les pressions à appliquer à un élément de surface de la fissure pour calculer le champ de contrainte singulier sont (§ 1.2.3):

$$
\mathrm{d} X=\tau_{x z} \mathrm{~d} S \quad \mathrm{~d} Y=\tau_{y z} \mathrm{~d} S \quad \mathrm{~d} Z=\sigma_{z z} \mathrm{~d} S .
$$

On voit que les contraintes qui ont leurs deux indices dans le plan de la fissure, les seules dont dépend la contrainte non singulière tangente au front de fissure, n'interviennent pas dans le champ singulier, dont la théorie linéaire admet qu'il suffit à décrire le phénomène. Dans le cas envisagé du cylindre, la contrainte circonférentielle est donc sans influence (Fig. 3).

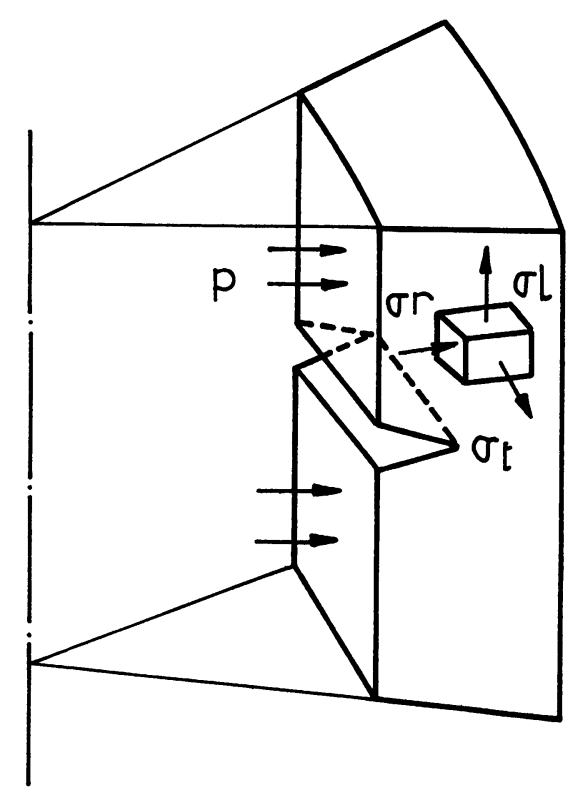

FIg. 3.
D’autre part, il est démontré (§ 1.2.2) que sur une fissure fermée ou dont le front est assez long, la zone singulière est en déformation plane.

On peut donc dans la théorie linéaire utiliser validement pour tous les problèmes de déformation plane le $K_{\mathrm{IC}}$ mesuré par les éprouvettes A. S. T. M. en déformation plane, quelle que soit la triaxialité des contraintes en l'absence de fissure.

Cependant, il ne faut pas s'attendre que la théorie linéaire, qui suppose le matériau idéalement continu du mécanicien, puisse rendre compte des phénomènes observés par le métallurgiste ou le physicien, par exemple de l'influence possible d'une contrainte finie dans le plan de la fissure sur la formation des clivages en avant du front de la fissure.

Jusqu'à présent, la dispersion des phénomènes n'a pas permis de mettre en évidence une variation significative du $K_{\text {IC }}$ avec la géométrie et le chargement.

1.5 Calcul des facteurs D'INTENSité De CONTRAINTE. - Jusqu'au début de 1973, on avait envisagé d'essayer de réunir en un formulaire, obligatoirement incomplet, les solutions des problèmes les plus importants. Le seul formulaire alors publié (Paris et Sih [23]) était un peu ancien. Cette situation a été profondément modifiée par la parution, dans l'été 1973, de deux formulaires plus modernes et plus complets, publiés l'un par G. C. Sih, l'autre par Tada, Paris et Irwin ; un troisième, d'origine anglaise, est annoncé.

On trouve dans ces ouvrages des exposés généraux sur les méthodes de calcul, souvent limités aux problèmes plans, et les solutions pour beaucoup de géométries et de chargements. Mais on constate que les seuls problèmes de révolution traités sont ceux de la barre circulaire entaillée et de la fissure circulaire, dite "penny shaped", dans un milieu indéfini. Aucun problème sur les cylindres de révolution creux n'est traité. Et pour les problèmes plans, si on sort des géométries étudiées, ce qui est fréquent pour les pièces de machines, il faut recourir aux méthodes numériques.

Les formulaires sont donc des monuments d'érudition qui donnent au spécialiste des informations de première valeur, mais que le bureau d'études ne peut que difficilement utiliser. Un nouveau formulaire aurait été très inférieur aux précédents et n'aurait pas comblé cette lacune.

On a donc préféré chercher à mettre au point une méthode de calcul numérique, et à retirer pour une géométrie donnée des résultats à partir desquels on calculerait simplement le facteur d'intensité de contrainte résultant de l'application à cette géométrie d'un chargement quelconque.

On a reporté aux chapitres 4 et 5 l'exposé de principe de la méthode retenue, et de quelques problèmes particuliers importants.

2. Limites de la théorie linéaire. Prolongements en domaine plastique. $-2.1 \mathrm{La}$ déformation plas- 
tique en fond de fissure existe toujours; Irwin et Orowan admettent comme expérimentalement démontré que l'extension de la fissure dissipe de l'énergie par déformation plastique.

Tant que la zone plastique reste petite, une approximation satisfaisante est la correction d'Irwin [17] qui prolonge la fissure d'une longueur :

$$
\begin{array}{ll}
r_{\mathrm{e}}=\frac{1}{2 \pi}\left(\frac{K_{\mathrm{I}}}{R_{\mathrm{e}}}\right)^{2} & \text { en contrainte plane } \\
r_{\mathrm{e}}=\frac{1}{6 \pi}\left(\frac{K_{\mathrm{I}}}{R_{\mathrm{e}}}\right)^{2} & \text { en déformation plane }
\end{array}
$$

avec (Fig. 4)

$$
a_{\mathrm{eff}}=a+r_{\mathrm{e}}
$$

valable tant que la loi de compliance corrigée :

$$
\delta l=C\left(a_{\text {eff }}\right) F
$$

reste pratiquement linéaire.
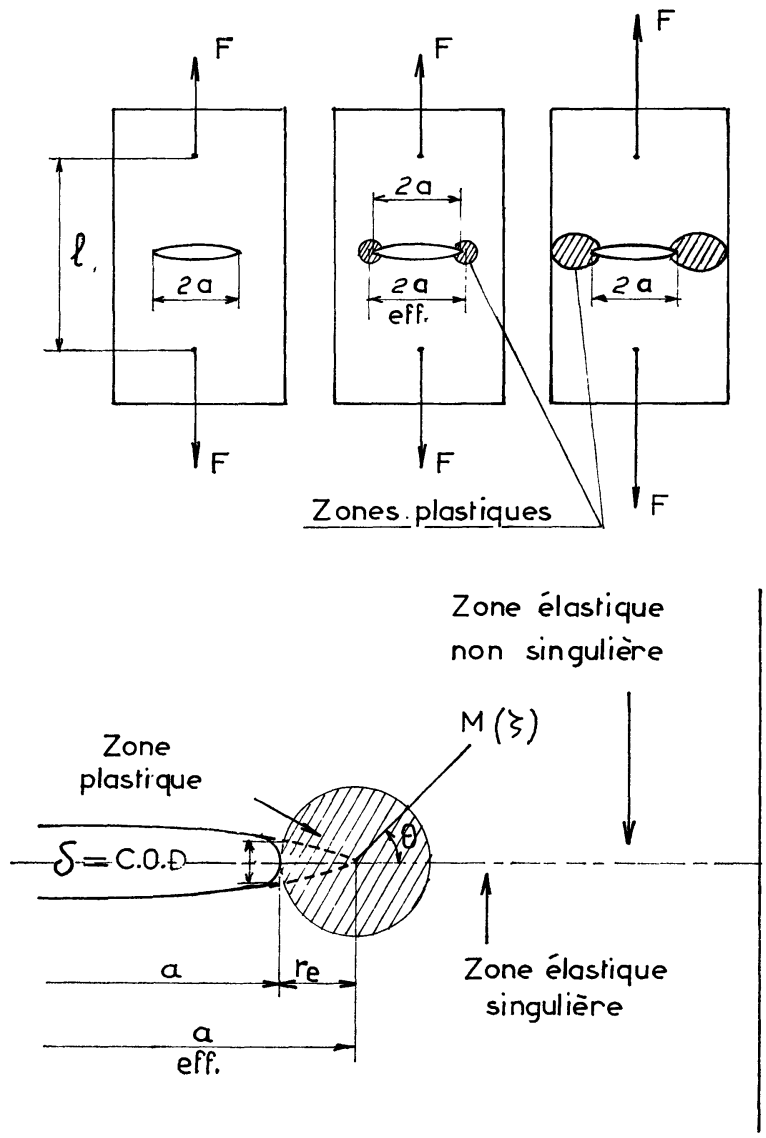

FIG. 4.

Alors, on trouve successivement en avant de la fissure (Fig. 4) :

- une petite zone plastique,

- une zone singulière élastique entourant la précédente, dans laquelle les contraintes sont définies par $K_{\mathrm{I}}$,

- une zone élastique non singulière.
Tant que cette configuration est réalisée, la théorie linéaire reste une approximation satisfaisante. Ceci exige des dimensions de pièce ou d'éprouvette croissant avec $\left(K_{\mathrm{I}} / R_{\mathrm{e}}\right)^{2}$ qui peut être grand pour les matériaux tenaces ayant une limite d'élasticité pas trop élevée, qu'on utilise dans les grandes constructions.

2.2 Dans les limites de la correction ci-dessus, l'ouverture en fond de fissure, au bord de la zone plastique, ou «crack opening displacement» (C. O.D.) est directement liée à $K_{\mathrm{I}}$ et peut être prise comme critère de rupture au même titre que $K_{\mathrm{I}}$.

Des travaux britanniques se sont attachés à montrer que ce critère pouvait encore être utilisé lorsque, la zone plastique devenant importante, la linéarité disparaît. Ces travaux n'ont pas été étudiés au cours de cette recherche.

Cependant, le calcul du C. O. D. pour une géométrie et un chargement quelconques, dans la zone de grandes déformations plastiques, est toujours difficile. Si le C. O. D. critique est un excellent critère de ténacité des aciers, il semble difficile de l'utiliser en mécanique hors des chargements simples.

Rice [24] a montré qu'une intégrale curviligne, dite $J$, indépendante du trajet d'intégration autour $\mathrm{du}$ front de fissure pouvait caractériser la partie très déformée de la zone plastique, et être retenue comme critère de rupture en domaine plastique. En calculant $J$ le long d'un trajet assez éloigné du front de fissure pour que les contraintes et déformations soient bien connues, on évite les difficultés de calcul du C. O. D. La valeur critique de $J$ est d'ailleurs :

$$
J_{\mathrm{IC}}=\mathcal{G}_{\mathrm{IC}} \text {. }
$$

La mise en œuvre de cette méthode est plus difficile qu'en domaine linéaire. En outre, $J$ n'est définie que pour les problèmes plans, et on n'a pas encore explicité l'intégrale qui devrait être utilisée pour les problèmes de révolution ou à trois dimensions.

2.3 Comme on l'a fait remarquer au paragraphe 1.4 à propos de la physique des décohésions, la déformation plastique peut faire remettre en cause l'universalité du $K_{\mathrm{IC}}$ mesuré sur les éprouvettes A. S. T. M. Ici encore on peut jusqu'à présent penser que cette universalité peut être admise à l'intérieur des dispersions observées.

Cependant, des expériences récentes (Auger et François [1]) ont relevé l'influence d'une pression hydrostatique élevée. On ne peut encore dire si ces expériences conduiront à admettre dans les problèmes usuels une influence pratique de la triaxialité des contraintes en l'absence de fissure.

2.4 Il semble aussi que la théorie linéaire puisse être utilisée pratiquement avec une approximation convenable au-delà de son domaine de validité. Il faudrait approfondir cette question afin de s'affranchir éventuellement de calculs plastiques difficiles. 
2.5 Une application intéressante de l'étude en domaine plastique est la mesure de $\mathcal{G}_{\mathrm{IC}}$, donc de $K_{\mathrm{IC}}$, par l'intermédiaire de $J_{\mathrm{IC}}$, sur de petites éprouvettes rompues en domaine plastique; une action de recherche concertée est en cours sur ce sujet.

3. Utilisation de la mécanique de la rupture hors du domaine de la rupture brutale. - Si le facteur d'intensité de contrainte a une réalité physique, il est naturel qu'on le trouve dans les problèmes de fissure, en dehors de la rupture brutale.

\subsection{LA LOI EMPIRIQUE DE PARIS :}

$$
\frac{\mathrm{d} a}{\mathrm{~d} N}=C_{0}(\Delta K)^{m}
$$

rend assez bien compte de la progression des fissures par fatigue au-dessous de la dimension critique.

La connaissance, même approximative, de $C_{0}$ et $m$, pour des constructions soumises à des chargements cycliques permettra d'estimer la progression de fissures non détectées. On pourra ainsi vérifier si des matériaux, intéressants par leurs caractéristiques de traction, ne présentent pas des dangers de ce fait, et retenir le meilleur compromis.

3.2 En corrosion sous contrainte, l'existence d'un seuil de $K_{\mathrm{I}}$, dit $K_{\mathrm{ICSC}}$, au-dessous duquel l'environnement est sans action, et au-dessus duquel une fissure progresse sous chargement statique, est connue.

La détermination de ce seuil permet l'estimation des dimensions des défauts qui peuvent devenir dangereux. On peut l'utiliser en Mécanique, alors que les essais classiques de rupture différée permettent seulement de classer les matériaux.

4. Méthode de calcul des facteurs d'intensité de contrainte. - 4.1 FONCTION D'INFLUENCE - FONCTION DE GREEN. - On considère pour un problème plan une fissure rectiligne, de longueur $l$, sur l'axe $o x$, dont le front $A$ a les coordonnées, $l, o$ (Fig. 5); on applique au point $x, o(x \leqslant l)$ la force $F$ répartie sur l'épaisseur $e$. On définit la fonction d'influence pour le $K_{\mathrm{I}}$ produit en $A(l, o)$ par $F$ appliquée en $(x, o)$ :

$$
G(l, x)=\frac{e}{F} K_{\mathrm{IF}}(l, x)
$$

$G$ et $K_{1 F}$ contiennent aussi les paramètres géométriques $\mathrm{du}$ solide fissuré.

Si on applique sur la fissure une pression répartie $p(x)$, problème auquel on ramène tout calcul de $K$ (§ 1.2.3) il est évident que la solution est :

$$
K_{\mathrm{I}}(l)=\int_{0}^{l} G(l, x) p(x) \mathrm{d} x .
$$

Si on connaît $G(l, x)$ le problème est ramené à une quadrature.

$G(l, x)$ peut être rattachée à la fonction de Green $\Gamma\left(\xi_{i}, x_{i}\right)(i=1,2)$ du problème plus général du $K_{\mathrm{I}}$

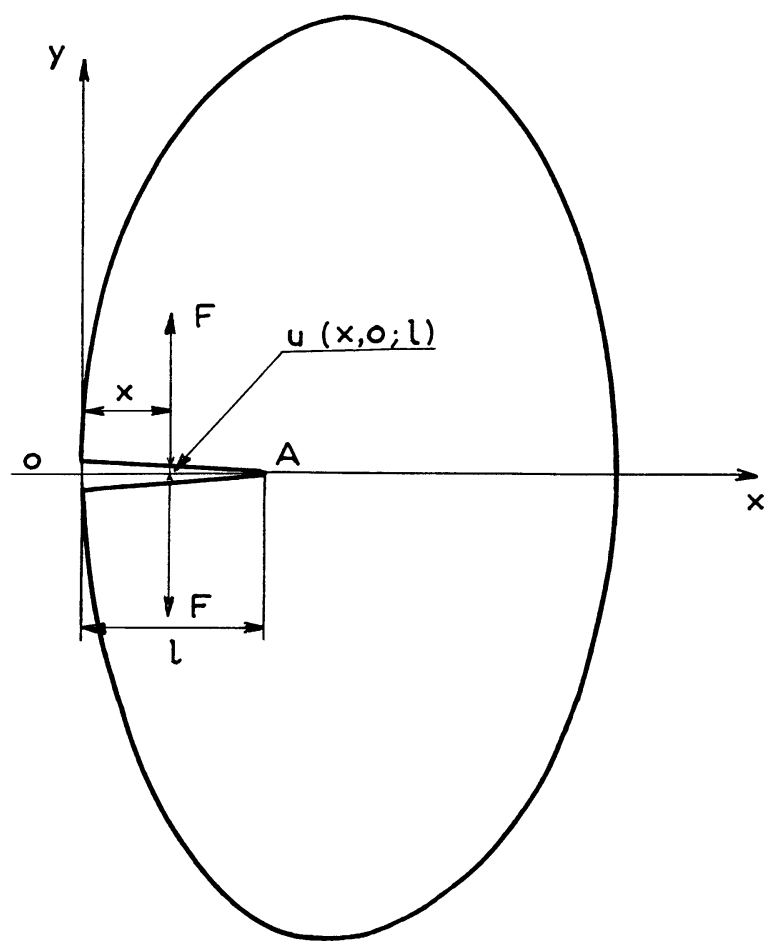

FIG. 5.

produit au point $\xi_{1,2}$ par la charge unité appliquée au point $x_{1,2}$. Souvent, on se limite comme ci-dessus à $\xi_{i}=l, o$ et $x_{i}=x, o$. Mais très souvent la dénomination trop générale de fonction de Green est donnée à la fonction d'influence $G(l, x)$. En fait, $l$ considéré comme une variable dans la fonction de Green est dans $G(l, x)$ un paramètre du corps fissuré.

La fonction d'influence peut être généralisée à un problème de révolution en remarquant une différence par rapport au problème plan: les surfaces $e \mathrm{~d} x$ et $e \mathrm{~d} l$ doivent être remplacées par :

$s_{F} \mathrm{~d} x=2 \pi\left(R_{i}+x\right) \mathrm{d} x$ pour l'application de $p(x)$ $s_{\mathrm{C}} \mathrm{d} l=2 \pi\left(R_{i}+l\right) \mathrm{d} l$ pour l'extension de la fissure. D'où la définition qui généralise (7)

$$
K_{\mathrm{I}}(l)=\int_{0}^{l} \frac{R_{i}+x}{R_{i}+l} G(l, x) p(x) \mathrm{d} x .
$$

La méthode est très générale, mais le petit nombre de fonctions d'influence explicitées pour des problèmes de fissures se rapporte à des géométries assez particulières, souvent peu utilisables par les Bureaux d'Etudes. En vue de calculs de $K$ sous contrainte thermique, Emery et al. [10] ont établi des courbes donnant numériquement de telles fonctions pour des bandes de longueur finie, de proportions variables. Il ne semble pas qu'il en ait été publié pour des problèmes de révolution, même simples, comme ceux relatifs aux cylindres.

Il est possible de donner une expression générale des fonctions d'influence pour les problèmes plans et de révolution. 
La force concentrée $F$ est répartie sur l'épaisseur $s_{F}=e$ pour un problème plan, et sur la circonférence $s_{F}=2 \pi\left(R_{i}+x\right)$ pour un problème de révolution.

On considère l'ouverture de la fissure au point d'application :

$$
v(l, x)=2 u(l, x)=C(l, x) . F
$$

et la variation d'énergie potentielle résultant de l'existence de la fissure et de la force $F$ :

$$
P(l, x)-P_{0}=-\frac{1}{2} F v=-\frac{F^{2}}{2} C(l, x) .
$$

Pour le calcul de $\mathfrak{S}$ on introduit la longueur du front de fissure :

$s_{\mathrm{c}}=e$

pour un problème plan

$s_{\mathrm{c}}=2 \pi\left(R_{i}+l\right)$ pour un problème de révolution .

D'où :

$\mathcal{G}=-\frac{1}{s_{\mathrm{c}}} \frac{\partial P}{\partial l}=\frac{F^{2}}{2 s_{\mathrm{c}}} \frac{\partial C(l, x)}{\partial l}$

$K=\left[\frac{E \mathcal{G}}{1-v^{2}}\right]^{1 / 2}=F\left[\frac{E}{2\left(1-v^{2}\right)} \cdot \frac{1}{s_{\mathrm{c}}} \cdot \frac{\partial C(l, x)}{\partial l}\right]^{1 / 2}$

et la fonction d'influence :

$$
G(l, x)=\frac{s_{\mathrm{c}}}{F} K=\left[\frac{E}{2\left(1-v^{2}\right)} \cdot s_{\mathrm{c}} \cdot \frac{\partial C(l, x)}{\partial l}\right]^{1 / 2}
$$

qui ne dépend que de la géométrie du solide fissuré. $C(l, x)$ étant différent pour un problème plan et un problème de révolution.

4.2 FonCtion DE POIDS. - Par la voie des équations intégrales singulières, Bueckner [7-9] est arrivé pour le problème plan à :

$$
K(l)=\sqrt{\frac{2}{\pi}} \int_{0}^{l} M(l, x) p(x) \mathrm{d} x
$$

et a appelé $M(l, x)$ fonction de poids ; celle-ci est strictement équivalente à la fonction d'influence ou à la fonction de Green, dans le sens restreint indiqué ci-dessus, et ne dépend que de la géométrie du solide fissuré ; le facteur $\sqrt{2 / \pi}$ fait que, au voisinage de $x=l$ :

$$
M(l, x)=[l-x]^{-1 / 2}+M^{*}(x) .
$$

Il est en pratique avantageux d'utiliser la fonction sans dimension, non singulière :

$$
\begin{aligned}
& m(x)=M(x)[l-x]^{1 / 2} . \\
& m(l)=1 .
\end{aligned}
$$

En posant $x / l=u$

$$
K(l)=\sqrt{\frac{2 l}{\pi}} \int_{0}^{1} p(l u) m(u)[1-u]^{-1 / 2} \mathrm{~d} u .
$$

Il peut être plus commode de prendre comme longueur de référence une longueur du solide non fissuré ; par exemple son épaisseur $W$ pour une plaque ou un cylindre de révolution. En posant $x / W=v$

$K(l)=\sqrt{\frac{2 W}{\pi}} \int_{0}^{l / W} p(W v) m(v)\left[\frac{l}{W}-v\right]^{-1 / 2} \mathrm{~d} v$.

Dans les fonctions $M$ et $m$ entrent les paramètres de géométrie :

$l$ : longueur de la fissure,

$\alpha_{i}$ : paramètres définissant le corps non fissuré,

$\beta_{j}$ : paramètres liant la fissure au solide.

Dans $p(x)$ entrent les paramètres du corps non fissuré ; ceux de la fissure n'y figurent que si la position de celle-ci a une importance.

Bueckner a donné les fonctions de poids pour une bande infinie, et les a mises sous la forme $(l / W<0,5)$

$$
m\left(\frac{x}{l} ; \frac{l}{W}\right)=1+m_{1}\left(1-\frac{x}{l}\right)+m_{2}\left(1-\frac{x}{l}\right)^{2}
$$

où $m_{1}$ et $m_{2}$ sont des polynômes en $l / W$.

Pour un problème de révolution, Bueckner a généralisé (15) par :

$$
K(l)=\sqrt{\frac{2}{\pi}} \int_{0}^{l} p(x) M(x) \frac{R_{i}+x}{R_{i}+l} \mathrm{~d} x .
$$

Pour le problème tridimensionnel d'une fissure plane quelconque, une fonction de poids à deux variables peut être définie, mais le problème est sensiblement plus difficile.

4.3 EXPRESSION DES FONCTIONS DE POIDS DONNÉE PAR RICE. - Rice [25] a donné une méthode de calcul des fonctions $M(x)$ ou $G(x)$. Si on considère un corps plan ou de révolution ayant une fissure rectiligne sur l'axe $O x(y=o)$ (Fig. 6), et soumis à un chargement quelconque $\mathrm{Cl}$, Rice a établi des relations équivalentes à (13) et (14) et a montré que, en déformation plane, la fonction :

$$
h(x, o ; l)=\frac{E}{2\left(1-v^{2}\right)} \frac{1}{K^{(1)}(l)} \frac{\partial u^{(1)}(x, o ; l)}{\partial l}
$$

où $u^{(1)}(x, o ; l)$ est le déplacement d'un point de la fissure ou demi ouverture, est indépendante du chargement et ne dépend que de la géométrie. En outre :

$$
h(x, o ; l) \equiv \frac{1}{\sqrt{2 \pi}} M(l ; x) \equiv \frac{1}{2} G(l, x) .
$$

Le théorème de Rice s'applique à des chargements très généraux; on se limite à ce cas particulier qui est en pratique suffisant pour les calculs de $K$.

La conséquence est que lorsqu'on fera sur une géométrie donnée le calcul du $K$ pour un chargement quelconque, éventuellement très simple, par une 

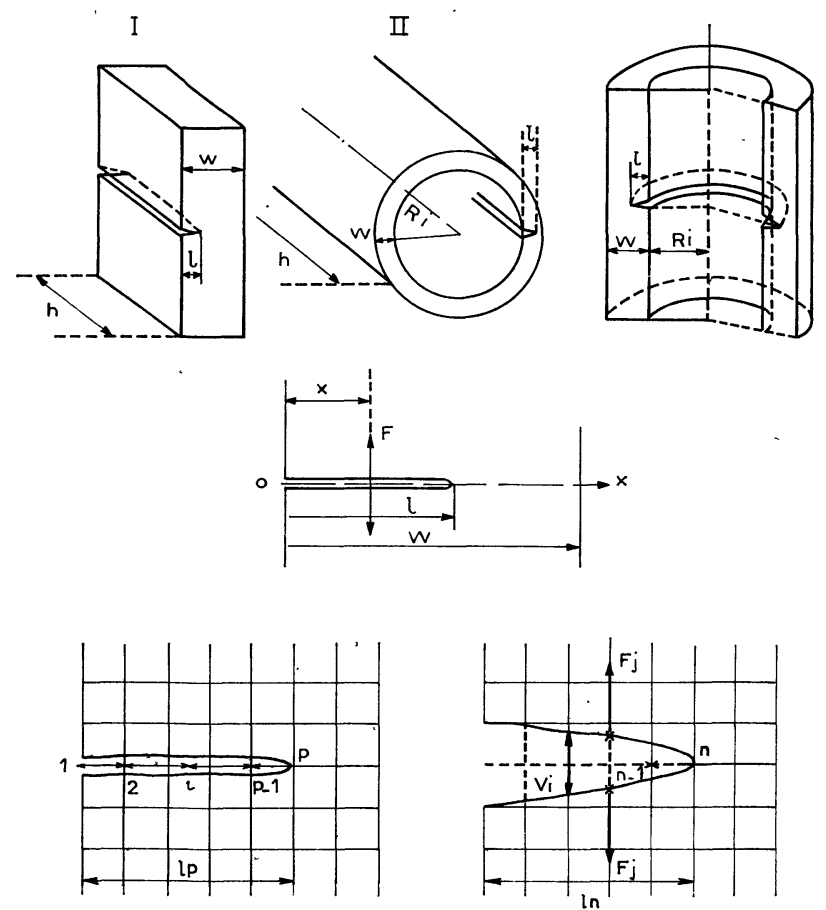

FIG. 6.

méthode algébrique ou numérique, si on sait aussi calculer $\partial u / \partial l$ sur la fissure, on obtiendra par le fait même la fonction d'influence ou la fonction de poids de Bueckner, attachée à cette géométrie, qui ramène à une quadrature le calcul du $K$ d'un chargement quelconque appliqué à cette géométrie.

$h(x, o ; l)$ est singulier comme $M(x)$; Rice a montré que sur la fissure;

$$
h(x, o ; l)=[2 \pi(l-x)]^{-1 / 2}+h^{*} .
$$

La généralisation au problème tridimensionnel d'une fissure plane quelconque est possible mais difficile.

4. 4 Les CONSÉQUenCES DE CES THÉORÈMES POUR UN CALCUL INDUSTRIÉL DES $K$ SONT IMPORTANTES. La connaissance des fonctions de poids enlèvera aux formulaires leur caractère de collection de solutions ingénieuses juxtaposées, parmi lesquelles il est souvent difficile de trouver la bonne formule, et réduira le calcul des $K$ à une quadrature à la portée des bureaux d'études. Le calcul des fonctions de poids reste cependant un problème de spécialistes.

Même si la fonction de poids est connue, le calcul des $K$ nécessite la connaissance préalable des contraintes qui règneraient dans le solide non fissuré à l'emplacement de la fissure; c'est un problème d'élasticité classique, qu'on peut résoudre, éventuellement par un calcul númérique, mais dont la solution n'est pas toujours simple.

4.5 La démonstration de Rice est limitée à des solides et des chargements symétriques par rapport au plan de la fissure, et suppose l'extension de celle-ci dans son plan. Il semble que l'essentiel soit que la fissure s'étende dans son plan, et pour que physiquement il puisse en être ainsi, il faut que le plan de la fissure soit dans le solide fissuré un plan de contraintes principales, donc sans cisaillement. Cela exige peut-être la symétrie.

4.6 CALCUL NUMÉRIQUE DeES-FONCTIONS DE POIDS. La méthode de Bụeckner conduit au calcul par les équations intégrales.

Disposant du programme d'élasticité par éléments finis Titus (Hasselin et al. [13]) on avait d'abord cherché à calculer $K$ par extrapolation de :

$$
\sigma_{r r}[2 \pi r]^{1 / 2} \text { ou } u_{y}\left[\frac{2 r}{\pi}\right]^{-1 / 2}
$$

ce qui est possible, mais assez long malgré l'utilisation d'éléments spéciaux à la pointe de la fissure. D'autre part, il fallait un calcul complet pour chaque chargement sur une géométrie donnée.

Pour éviter ces inconvénients, et ne faire qu'un calcul par géométrie, on a envisagé de calculer par éléments finis la fonction $h(x, o ; l)$ de Rice en calculant pour un chargement simple $K_{\mathrm{I}}(l)$ et sur la fissure $\partial u(x ; l) / \partial l$; la remarque (21) sur la singularité de $h(x, o ; l)$ éliminait toute difficulté au voisinage du front de fissure.

On a ensuite préféré calculer la fonction d'influence $G(l ; x)(14)$, strictement équivalente à la fonction de Rice et à la fonction de poids de Bueckner.

Sur une fissure de longueur $l^{p}$, on applique des forces $F_{i}$ aux nœuds $i$, et on calcule la variation d'énergie potentielle (10), puis $\mathcal{G}$ (12) en fermant progressivement la fissure à partir de sa longueur maximale (Fig. 6). On en tire la fonction d'influence (14) ou la fonction de poids.

Dans le formalisme des éléments finis :

$$
v_{i}\left(l^{p}, x_{i}\right)=C_{i i}\left(l^{p}, x_{i}\right) F_{i}
$$

et on arrive à :

$$
K_{\mathrm{I}}^{p}=\left[\frac{E}{2\left(1-v^{2}\right)}\right]^{1 / 2} \sum F_{i}\left[\frac{1}{s_{\mathrm{c}}} \frac{C_{i i}^{p}-C_{i i}^{p-1}}{l^{p}-l^{p-1}}\right]^{1 / 2} .
$$

Par identification 'aux définitions (7) et (8) de la fonction d'influence (15) et (18) de la fonction de poids, on voit facilement que les longueurs $S_{F_{i}}$ d'application des forces $F_{i}$ s'éliminent, et on obtient :

$$
\begin{aligned}
M\left(x_{i}, l^{p}\right) & =\sqrt{\frac{\pi}{2}} G\left(l^{p}, x_{i}\right) \\
& =\frac{1}{2}\left[\frac{\pi E}{1-v^{2}} S_{\mathrm{c}} \frac{C_{i i}^{p}-C_{i i}^{p-1}}{l^{p}-l^{p-1}}\right]^{1 / 2}
\end{aligned}
$$

$s_{\mathrm{c}}=e \quad$ pour un problème plan

$s_{\mathrm{c}}=2 \pi\left(R_{\mathrm{int}}+l\right)$ pour un problème de révolution. 
On sort directement de l'ordinateur la fonction sans dimension non singulière :

$$
m\left(x, l^{p}\right)=M\left(x, l^{p}\right)\left[l^{p}-x\right]^{1 / 2} .
$$

4.7 Pour VÉRIFIER LA VALIDITÉ DE LA MÉTHODE, ON L'A APPLIQUÉE D'ABORD A DES PROBLÈMES DÉJA RÉSOLUS.

- bande infinie soumise à une traction uniforme :

- calcul de $K$; différence avec les solutions de Gross et Bowie (Paris et Sih, [23]) inférieure à $2 \%$ (Fig. 7) ;

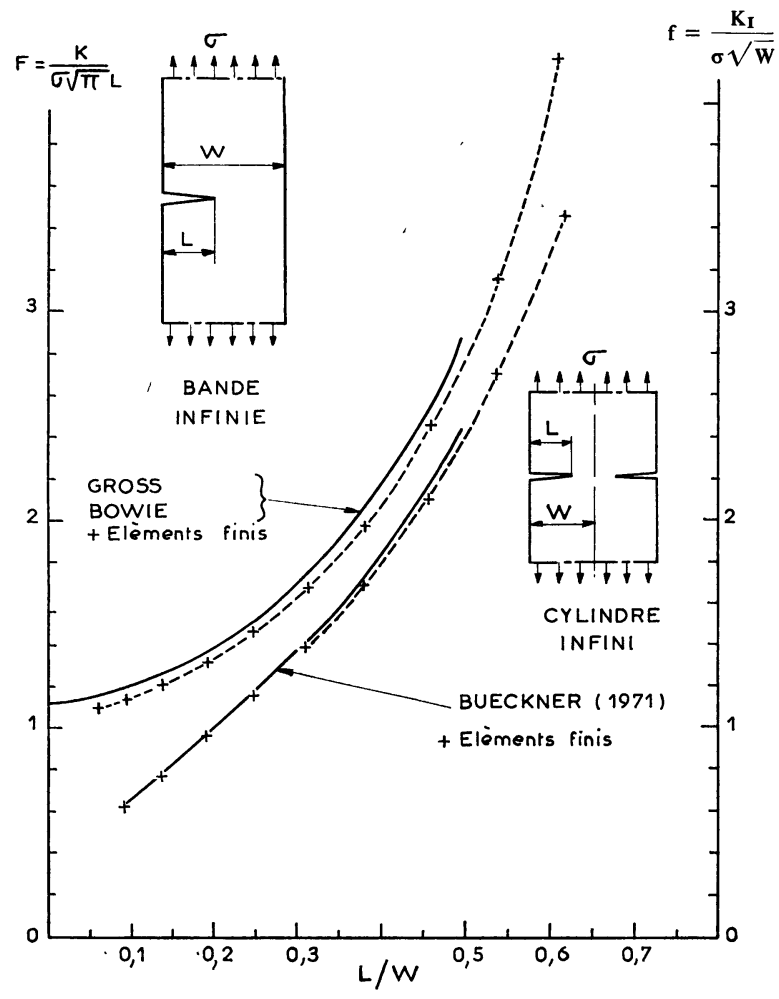

FIG. 7.

calcul des fonctions poids, identité pratique avec les valeurs données par" Bueckner [9] (Fig. 8) ;

- anneau fissuré soumis à une pression appliquée sur la paroi interne et les faces de la fissure, calcul de $K$ et comparaison avec une solution de Bowie [5] ; bonne coïncidence (Fig. 9) ;

- barre ronde ayant une fissure circonférentielle extérieure soumise à une traction uniforme, calcul de $K$ et comparaison avec la solution de Bueckner [8] ; voir aussi Paris et Sih [23], discussion; très bonne coïncidence (Fig. 7).

Ces vérifications permettent d'avoir confiance dans la méthode et le programme mis au point. On a donc calculé les fonctions de poids de deux géométries importantes en pratique ;

- longue fissure axiale (anneau fissuré) (Fig. 10) ;

- fissure circonférentielle complète (Fig. 11) ;

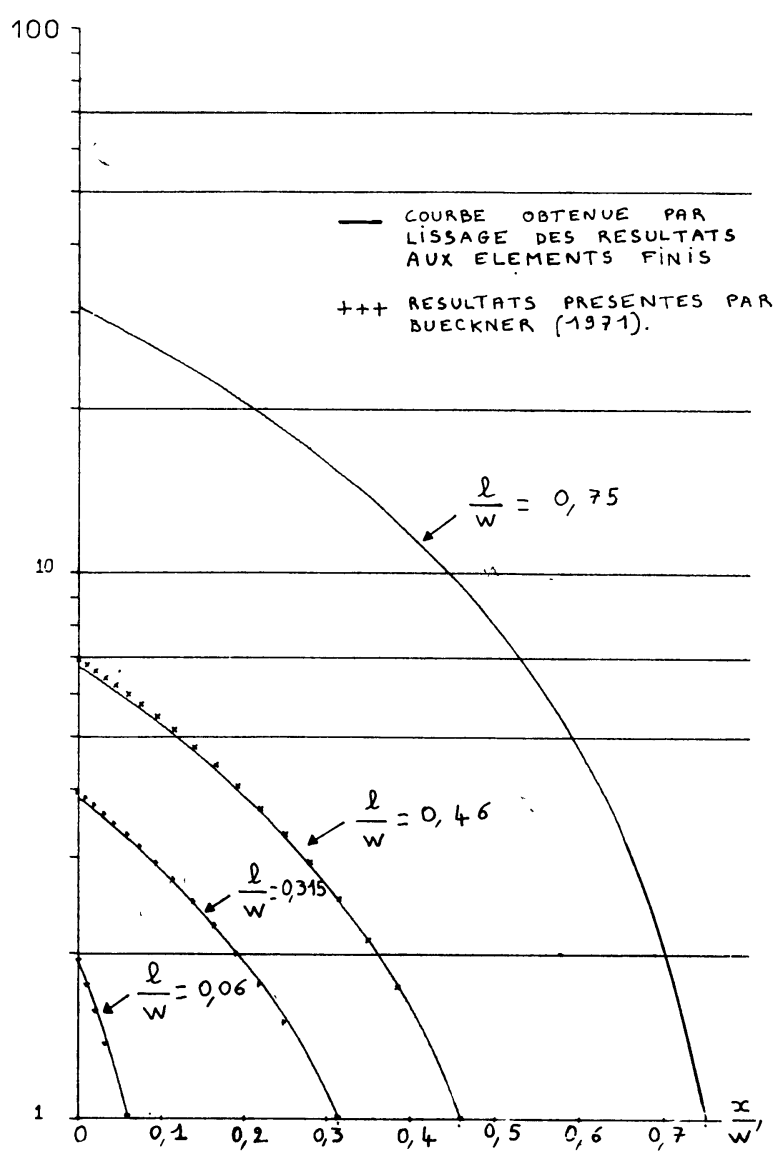

FIG. 8. - Fonction $m\left(\frac{x}{W}, \frac{l}{W}\right)$ pour la bande infinie.

pour lesquelles, faute de solution, on faisait souvent l'approximation pessimiste du problème plan (Fig. 8).

Ces fonctions de poids permettent de calculer par une quadrature le $K$ résultant de n'importe quelle distribution de contrainte sur la fissure.

Pour une longue fissure axiale dans un cylindre :

$$
K=\sqrt{\frac{2 W}{\pi}} \int_{0}^{l / W} \frac{m\left(\frac{x}{W} ; \frac{l}{W}, \frac{R_{i}}{W}\right)}{\left[\frac{l}{W}-\frac{x}{W}\right]^{1 / 2}} \sigma\left(\frac{x}{W} ; \frac{W}{R_{i}}\right) d\left(\frac{x}{W}\right)
$$

et pour une fissure circonférentielle :

$$
\begin{aligned}
K=\sqrt{\frac{2 W}{\pi}} \int_{0}^{l / W} \frac{R_{i}+x}{R_{i}+l} \frac{m\left(\frac{x}{W} ; \frac{l}{W}, \frac{R_{i}}{W}\right)}{\left[\frac{l}{W}-\frac{x}{W}\right]^{1 / 2}} \times \\
\times \sigma\left(\frac{x}{W} ; \frac{W}{R_{i}}\right) \mathrm{d}\left(\frac{x}{W}\right) .
\end{aligned}
$$

5. Remarques sur d'autres problèmes de calcul de facteurs d'intensité de contrainte. - 5.1 Contraintes thermiQues. - Lorsque la fissure est 
CYLINDRE INF,

FISSURE AXIALE

A UNE PRESSION I, pour $\frac{R i}{W}=2$
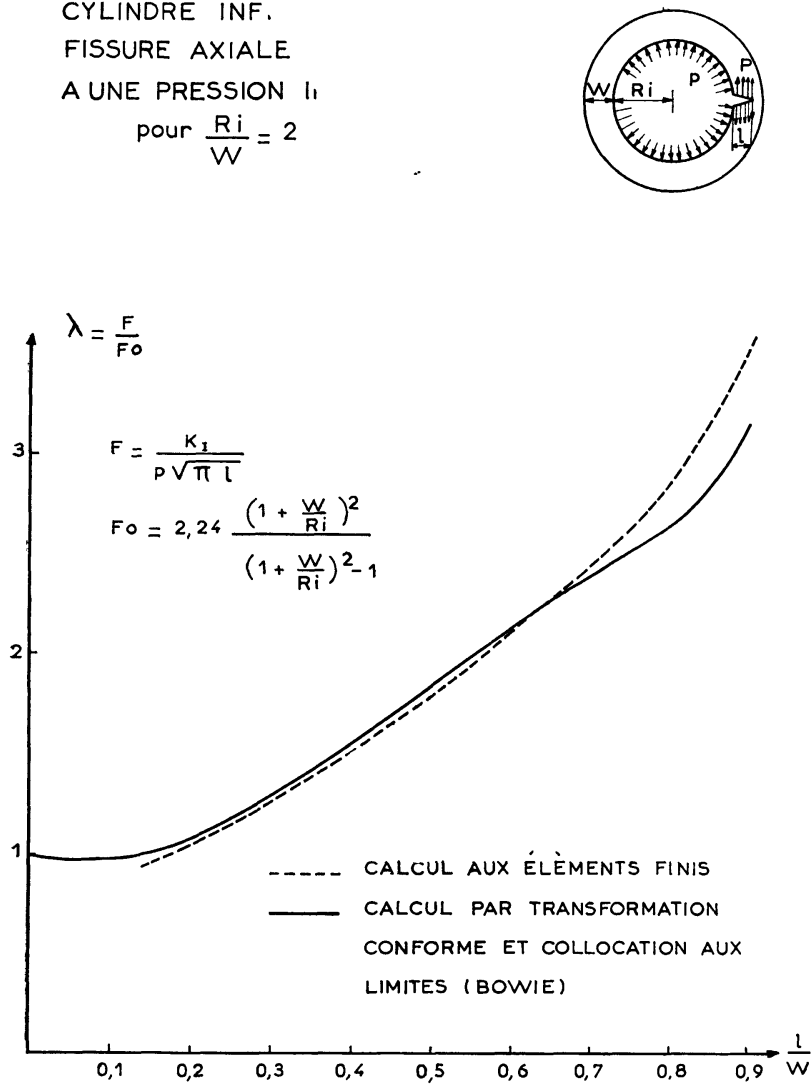

Fig. 9. - Cylindre infini avec fissure axiale soumis à une pression intérieure $p$ pour $R_{i} / W=2$.

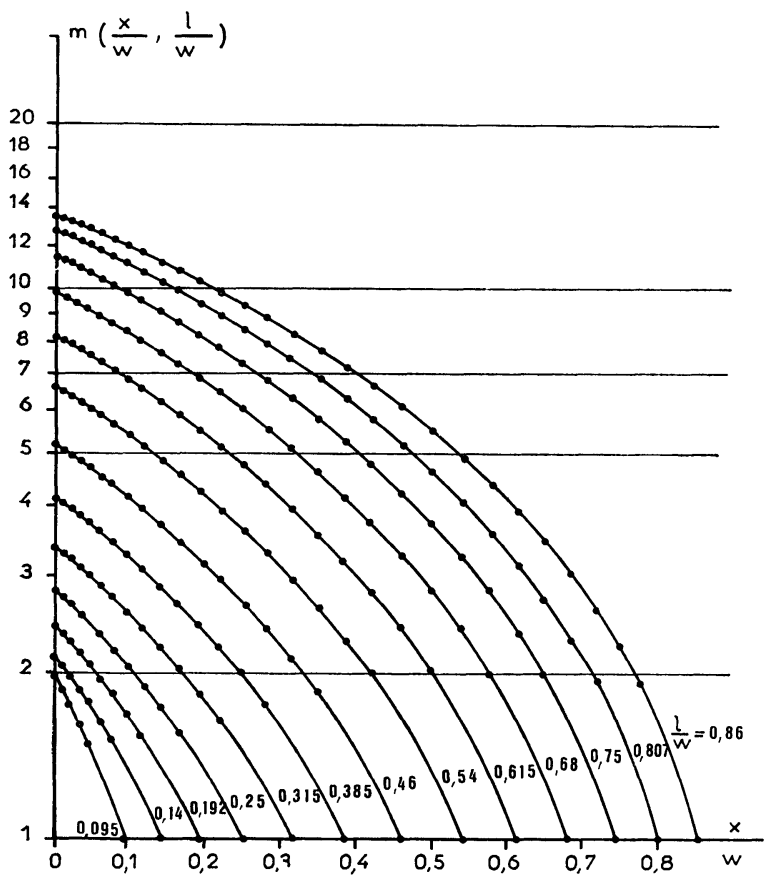

FIG. 10. - Fissure longitudinale $\frac{R}{W}=5$. Fonction $m\left(\frac{x}{W}, \frac{l}{W}\right)$

$$
K=\sqrt{\frac{2 W}{\pi}} \int_{0}^{l / W} \frac{m\left(\frac{x}{W}, \frac{l}{W}\right) \sigma\left(\frac{x}{W}\right)}{\sqrt{\frac{l}{W}-\frac{x}{W}}} \mathrm{~d} \frac{x}{W} .
$$

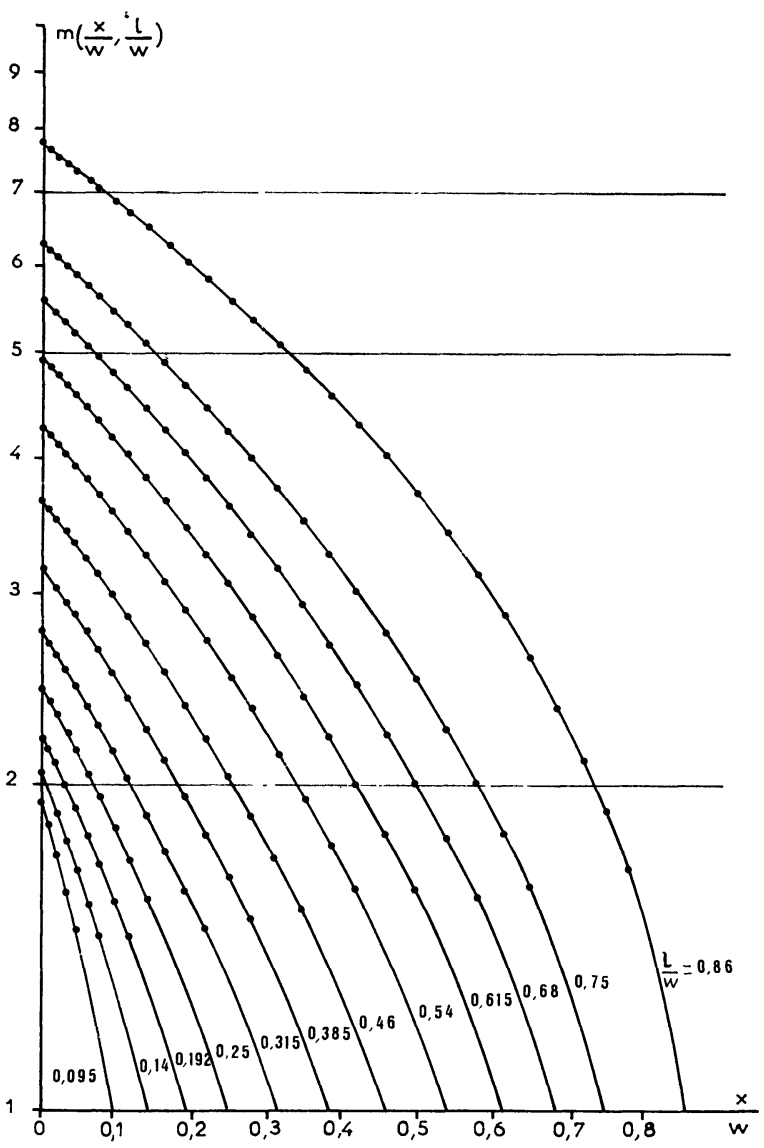

Fig. 11. - Fissure circonférentielle $\frac{R}{W}=5$. Fonction $m\left(\frac{x}{W}, \frac{l}{W}\right)$

$$
K=\sqrt{\frac{2}{2}-\frac{\dot{W}}{\pi}} \int_{0}^{l / W} \frac{R_{i}+x}{R_{i}+l} \frac{m\left(\frac{x}{W}, \frac{l}{W}\right) \sigma\left(\frac{x}{W}\right)}{\sqrt{\frac{l}{W}-\frac{x}{W}}} \mathrm{~d} \frac{x}{W}
$$

paradlèle au flux thermique, celui-ci n'étant pas perturbé par la fissure il n'y a aucune difficulté à appliquer la méthơde générale du chargement équivalent.

Le problème est apparemment moins simple lorsque la fissure introduit une anomalie de conductivité qui perturbe le flux. En introduisant dans le solide non fissuré la même anomalie sans altérer la continuité mécanique, on calcule un champ non singulier de températures, mais dont le flux peut être singulier. Les contraintes thermiques qui en résultent ne sont pas singulières (Sih [26]). En les appliquant sur la fissure, on est ramené au problème ordinaire du calcul de $K$. On résoud ainsi facilement les problèmes plans et de révolution; mais le problème d'un gradient thermique variable suivant l'épaisseur et d'une fissure traversante est un problème tridimensionnel dont la solution reste difficile.

5.2 Structures hyperstatiques. - Le cas le plus fréquent est celui des charpentes hyperstatiques, composées de barres plus ou moins complexes, soumises à des tractions et des moments qui dépendent des déformations. 
Il n'y a pas de difficulté de principe, mais :

- si les barres ont une section assez complexe, le calcul de $K$ sous une traction et un moment peut être difficile, et $K$ n'est pas toujours le critère de rupture ;

- la flexibilité des barres varie lorsque la fissure progresse et il faut en tenir compte dans le calcul des forces et moments à appliquer.

5.3 Les coques minces fissurées sur toute leur épaisseur sont un problème pratique important pour les appareils à pression et d'autres applications; une fuite met souvent l'appareil hors d'usage, mais est beaucoup moins grave qu'une rupture brutale. S'il y a une flexion de quelque importance, le problème est tridimensionnel et difficile. Des contradictions sont apparues dans des calculs utilisant la théorie de Kirchhoff ; elles ont été levées dans certains problèmes particuliers ; Bergez et Radenkovic [3-4] ont montré la validité et la généralité des résultats obtenus avec la théorie de Reissner. Il serait important pour les appareils à pression de comparer les valeurs numériques auxquels mènent ces résultats à celles établies pour les cylindres et sphères par Erdogan et Kibler [11] avec la théorie de Kirchhoff.

6. Conclusion. Suggestions pour d'autres recherches. - On est arrivé ainsi à donner une réponse aux questions qu'on se posait en 1971 , et on a donné une méthode pratique de calcul des fonctions de poids attachées à une géométrie donnée, dont la connaissance ramène tout calcul de $K$ à une quadrature.

Comme prolongement des résultats acquis, on peut envisager les problèmes mécaniques suivants :

- calculs en domaine élastique-plastique avec une déformation plastique étendue en avant du front de fissure ; domaine dans lequel le calcul élastique peut être prolongé avec une approximation pratiquement suffisante ;

- étude du passage du problème bidimensionnel (fissure longue ou circulaire) au problème tridimensionnel d'une fissure plus courte ; possibilité de trouver des coefficients approchés de correction;

- étude de la validité pratique du critère approché $K_{\max }=K_{\mathrm{IC}}$ lorsque $K$ est variable sur le contour de la fissure ;

- recherche de la formulation d'un critère général de rupture sous un chargement complexe.

Remerciements. - Les auteurs remercient Monsieur l'Ingénieur Général du Génie Maritime de Leiris et Monsieur le Professeur D. Radenkovic, dont les observations leur ont été précieuses.

\section{Bibliographie}

[1] Auger, J. P. et François, D., Mesures de ténacité d'un alliage d'aluminium à haute résistance sous pression hydrostatique, Revue Phys. Appl. 9 (1974) 639.

[2] Barenblatt, G. I., The Mathematical Theory of Equilibrium Cracks in Brittle Fracture, Adv. Appl. Mechanics 7 (1962).

[3] Bergez, D. et Radenkovic, D., La Caractérisation des Fissures dans les Plaques Fléchies et les Coques, $C$. $R$. Hebd. Séan. Acad. Sci. 17/VII/72 Série A (1962) 221.

[4] Bergez, D. et Radenkovic, D., On the Definition of StressIntensity Factors in Cracked Plates and Shells, 2nd Int. Conf. on Pressure Vessel Technology, San Antonio (A. S. M. E.) (1973) 1089-1094.

[5] BowiE, O. et Freese, C. E., Elastic Analysis for a radial crack in a circular ring, Eng. Fracture Mechanics, juin 1972.

[6] Bueckner, H. F., The Propagation of Cracks and the Energy of Elastic Deformation. Transactions of the A. S. M. E. (1958) 1225-1230.

[7] Bueckner, H. F., A novel Principle for the Computation of Stress-Intensity Factors, Z. Angew. Math. Mech. (1970) 529.

[8] BueCKNER, H. F., Weight Functions for the notched Bar, Z. A. M. M. (1971) 97-102.

[9] BueCKNER, H. F., Fields singularities and related integral Representations, chapitre V de Methods of Analysis and Solutions of cracks problems (édité par G. C. Sih-Nordhoff).

[10] Emery, A. F. et al., A Green's Function for the StressIntensity Factors of Edge Cracks and its Application to Thermal Stresses, J. of Basic Engineering (1969) 618.
[11] Erdogan, F. et Kibler, J. J., Cylindrical and Spherical Shells with Cracks, Intl. J. of Fracture Mech. 1969, 229

[12] Griffith, A. A., The Phenomena of Rupture and Flow in Solids. Philosophical Transactions of the Royal Society of London, Serie A 221 (1920) 163.

[13] Hasselin, G., Heliot, J. et Vouillon, C., L'utilisation du Code aux Eléments finis pour les Structures Nucléaires et l'Analyse des Contraintes internes, 2nd Int. Conf. on Structure Mechanics in Reactor Technology, Mémoire M 2/3, Berlin, 1973.

[14] IrwIN, G. R., Fracture Dynamics. Dans Fracturing of Metals. Am. Soc. for Metals (1948) 147.

[15] IRwin, G. R., Relation of Stresses near a Crack to the Crack Extension Force. Int. Congress on Applied Mech. Bruxelles 1956, page 245.

[16] IRwin, G. R., Analysis of Stresses and Strains near the end of a Crack traversing a Plate, J. Appl. Mech. (1957) 361.

[17] Irwin, G. R., Plastic Zone near a Crack and Fracture Toughness. Seventh Sagamore Ordnance Materials Research Conference, 1960.

[18] IRwin, G. R., Crack Extension Force for a part-through Crack in a Plate, J. Appl. Mech. (1962) 651.

[19] Kassir, M. K. et Sir, G. C., Three-dimensional Stress Distribution around an Elliptical Crack under Arbitrary loadings, J. Appl. Mech. (1966) 601.

[20] De LeIRIS, H., Triaxialité de contraintes et critères de nonfragilité. Association Technique Maritime et Aéronautique.

[21] De LeIrIs, H., Les Ruptures par Décalottage dans les Récipients sous pression à corps cylindrique, Sciences et Techniques de l'Armement (1970), p. 167 à 183, fasc. 1. 
[22] Orowan, E., Fundamentals of Brittle Behavior in Metals. Dans Fatigue and Fracture of Metals (édité par W. M. Murray) 1950, p. 139.

[23] Paris, P. C. et Sir, G. C., Stress Analysis of Cracks, dans ASTM-STP 381, Fracture Toughness and its Applications (1964), p. 30.

[24] Rice, J. R., A path independent integral and the approximate analysis of strain concentrations by notches and cracks, J. Appl. Mech. (1968) 379, 386.

[25] Rice, J. R., Some Remarks on Elastic Crack-Tip Stress Fields, Int. J. Solids Structures (1972) 751.
[26] SiH, G. C., Heat Conduction in the infinite Medium with Lines of Discontinuities, J. of Heat Transfer (1965) 293, 98.

[27] SiH, G. C., Handbook of Stress Intensity Factors. Ed. Institute of Fracture and Solid Mechanics, Lehigh University, Bethlehem, Pa., 18015.

[28] Tada, H., Paris, P. C. et Irwin, G. R., The Stress Analysis of Cracks Handbook. Ed. : Del Research Corporation, 427 Main Street, Hellertown, Pa., 18055. 